\title{
A Biologically Inspired Ratchet Model of Two Coupled Brownian Motors
}

\author{
Debasis Dan and A. M. Jayannavar \\ Institute of Physics, Sachivalaya Marg, Bhubaneswar 751005, India \\ Gautam I. Menon \\ The Institute of Mathematical Sciences, \\ C.I.T. Campus, Taramani, Chennai 600 113, India
}

\begin{abstract}
A ratchet model for coupled Brownian motors, inspired by the motion of individual two-headed molecular motors on cytoskeletal filaments, is proposed. Such motors are modeled as two elastically coupled Brownian particles, each of which moves in a flashing ratchet potential. The ratchet potentials felt by the individual particles are anti-correlated, modeling the successive binding and unbinding of the two motor heads to the filament. We obtain, via Langevin simulations, steady-state currents as functions of noise strength, the equilibrium separation of the particles and the rate of switching between potential states. We observe an enhanced current due to coupling, noise induced stability and phase-locked behaviour in the deterministic regime. A qualitative understanding of these features is provided.
\end{abstract}

Key words: ratchet, molecular motor, Langevin equation.

PACS: 87.16.Nn, 05.40.-a

\section{Introduction}

Motor proteins (kinesins, dyneins and myosins) are versatile biomolecules which shuttle cargo encapsulated in vesicles to different parts of the cell. Such transport occurs via the cytoskeleton, a cell-spanning polymeric network of microtubules, actin filaments and intermediate filaments. Motor proteins are

Email addresses: dan@iopb.res.in, jayan@iopb.res.in (Debasis Dan and A. M. Jayannavar), menon@imsc.ernet.in (Gautam I. Menon). 
also important ingredients of the mechanisms of muscle contraction and cell division. The quest for physical principles that enable such tiny molecular machines to function efficiently in a highly Brownian regime is a subject of ongoing interest.[1]

Molecular motors are typically dimeric[2]. Conventional kinesins, for example, consist of two identical proteins, each of which has a motor domain (the head) and a cargo binding domain. A stalk intervenes between the head and the cargo binding domain. The two stalks coil around each other to produce the dimer. The two heads walk unidirectionally along a micro-tubule, a long and fairly stiff polymer comprised of an asymmetric repeating unit, in 8 nm steps. Each such step is coupled to the hydrolysis of a molecule of ATP. Typically, such kinesins can walk several hundred steps before detaching from the micro-tubule track.

Simple models for molecular motors idealize them as Brownian motors $[1,3]$. A Brownian motor is a point particle with an overdamped equation of motion, which exhibits directed motion when subject to thermal and athermal noise[1,4]. The thermal noise is assumed to be Gaussian and satisfies a fluctuation-dissipation relation. For molecular motors, the athermal noise reflects the non-equilibrium driving of the motor through the irreversible hydrolysis of ATP.

The interaction of the motor with the molecular track is modeled in terms of an asymmetric periodic potential felt by the motor[1]. This potential switches between two or more states. The energy release upon ATP hydrolysis is believed to alter the coupling of the motor protein to the substrate. It is thus modeled by a switch in the potential state seen by the Brownian particle.

The fact that the motor molecule is a two-headed object is ignored in these simple models (however, see Refs. [5,6]). Experimentally, two structural elements of the junction of the two heads appear to be crucial to rapid motility - the neck and the neck linker. The neck linker lies just beyond the catalytic core of the head, while the neck leads from the neck linker to the coiled stalk. The coupling of the two heads via the neck region ensures that an elastic interaction operates between the heads. The enhanced processivity and rate of movement in $N$. Crassa conventional kinesins has been attributed, in part, to the enhanced flexibility of its neck region[7,8].

Models of motor activity also indicate that the binding of one head as a consequence of ATP hydrolysis correlates closely with the loosening of the other head upon the subsequent release of the inorganic phosphate[9]. This suggests that a more physically realistic Brownian motor model of the working of a two-headed molecular motor would involve (a) the incorporation of an elastic interaction between motor heads and (b) switching between potential states which are fully anti-correlated between heads, so that one head is loosely bound when the other is tightly bound and vice versa.

To develop these ideas further, we propose and study a simple model of a two-headed molecular motor interacting with a thermal bath at temperature $T$. Our model system consists of two elastically coupled overdamped Brownian 
particles moving in periodic potentials $V_{1}(x)$ and $V_{2}(x)$. These potentials alternate in time; we will assume this switching to be time periodic with period $\tau$. We take $V_{1}(x)$ to be a periodic asymmetric potential and $V_{2}(x)$ to be a flat potential. The potentials felt by the two particles are out of phase i.e. when one of them moves under the influence of potential $V_{1}(x)$, the other only sees the force due to the elastic linkage with the other particle.

Our model has some similarities, and yet some important differences, with others proposed earlier. Unlike the models proposed by Klumpp et. al. [5], and by Ajdari[6], our model has no multiplicative noise terms, although the time-periodic variation of the potential does enter multiplicatively in our equations of motion. Also, in our model, the potentials $V_{1}$ or $V_{2}$ felt by one head are (anti-)correlated with the potentials felt by the other, not uncorrelated as in earlier work.

We have studied our model numerically via Langevin simulations in the weak and strong coupling regimes. Our model exhibits a range of unusual behaviour, including an enhanced current due to coupling in some parameter range (this has been noted earlier in a different but related model [5]), drift in the deterministic regime (with phase-locked behaviour [11]), and noise induced stability [12]. Subsequent sections of this paper describe these features.

In the next section we propose the system of coupled Langevin equations to describe the model. In Sec. 3.1 we present results for our model in the deterministic regime, varying the coupling constant, the equilibrium separation of the two particles and the frequency of potential fluctuation. In Sec. 3.2 we discuss the behaviour of the current in the presence of thermal noise. Our conclusions are contained in Sec. 4.

\section{Model}

We consider two overdamped Brownian particles, coupled via an elastic interaction, moving in a two state flashing ratchet potential which switches between $V_{1}(x)$ and 0 periodically. The particles are in contact with a thermal bath at temperature $T$. Let $x_{1}(t)$ and $x_{2}(t)$ be the coordinates of the two particles. They are coupled through a spring of spring constant $k$ and equilibrium length $a$. The Langevin equations which governs our system are

$$
\begin{aligned}
& \gamma \dot{x_{1}}=-z_{1}(t) V^{\prime}\left(x_{1}\right)+k\left(\left(x_{1}-x_{2}\right)-a\right)+\xi_{1}(t) \\
& \gamma \dot{x_{2}}=-z_{2}(t) V^{\prime}\left(x_{2}\right)-k\left(\left(x_{1}-x_{2}\right)-a\right)+\xi_{2}(t)
\end{aligned}
$$

where $\xi_{i}(t)$ are Gaussian random forces with zero mean and a correlation given by $<\xi_{i}(t) \xi_{j}\left(t^{\prime}\right)>=2 D \delta_{i j} \delta\left(t-t^{\prime}\right)$. Here, $D=\frac{k_{B} \gamma T}{m}$. The $z_{i}$ 's are periodic functions with period $\tau$, given by $z_{1}(t)=1, z_{2}(t)=0$ for $0 \leq t<\frac{\tau}{2} ; z_{1}(t)=$ $0, z_{2}(t)=1$ for $\frac{\tau}{2} \leq t<\tau$. The asymmetric periodic potential with unit period 
is $V(x)=-\frac{U}{2 \pi}(\sin (2 \pi x)+0.25 \sin (4 \pi x))$. We work with dimensionless units wherever possible.

We solve these coupled equations numerically by Huen's method [13] and calculate the current in the asymptotic regime. The current $j$ is given by $j=\left\langle\frac{x(t)-x\left(t_{0}\right)}{t-t_{0}}\right\rangle$, where $<. .>$ denotes the ensemble average and $t_{0}$ the asymptotic time. We take $t_{0}=50 \tau, t=4000 \tau$ and average over 3000 ensembles. In the limiting case of rigid rod like coupling (i.e., $x_{1}-x_{2}=a$ ), we solve the associated Fokker-Planck equation $\frac{\partial P(x, t)}{\partial t}=-\frac{\partial J(x, t)}{\partial x} \quad[14]$

$$
\frac{\partial P(x, t)}{\partial t}=-\frac{\partial}{\partial x}\left(V^{\prime}(x)+\sqrt{D} \frac{\partial}{\partial x}\right) P(x, t)
$$

for $n \tau<t \leq(n+1 / 2) \tau$,

$$
\frac{\partial P(x, t)}{\partial t}=-\frac{\partial}{\partial x}\left(V^{\prime}(x+a)+\sqrt{D} \frac{\partial}{\partial x}\right) P(x, t),
$$

for $(n+1 / 2) \tau<t \leq(n+1) \tau$. Here $x$ is the position of one of the particle, the position of the other particle being fixed at $x+a$ and $P(x, t)$ is the probability density. The average current $j=\lim _{t \rightarrow \infty} \frac{1}{\tau} \int_{t}^{t+\tau} d t \int_{0}^{1} J(x, t) d x$. We have obtained $j$ by numerically solving the Fokker-Planck equation.

\section{Results and Discussions}

\subsection{Deterministic Current $(D=0)$}

A single particle moving in a flashing potential such as the one described above, exhibits no directional motion in the absence of thermal fluctuations. However, such motion can be induced in a system of elastically coupled particles using the compressibility of the spring [6,5]. If the equilibrium separation of the two particles is smaller than the length of the smaller arm of the potential $V(x)\left(L_{\min }=0.38\right)$ or larger than the longer arm $\left(L_{\max }=0.62\right)$, then the phase space of the system is a closed orbit. No directed current is possible for any value of $\tau$ or $k$. The window $L_{\max }-L_{\min }$, for which a directed current is obtained depends on the value of $\tau$ (Fig.(1A)). For smaller values of $\tau$, the length of the window for nonzero current is small, as the particle has less time to traverse to the basin of attraction of the next well. In Fig.(1B) we plot the current $j$ as function of $k$ for $a=0.5$, for different values of $\tau$. Observe that the current exhibits a phase locked behaviour, with step size becoming larger for smaller $\tau$ as observed previously in many different sys-

tems. Such phase locking arises in deterministic systems due to the interplay 

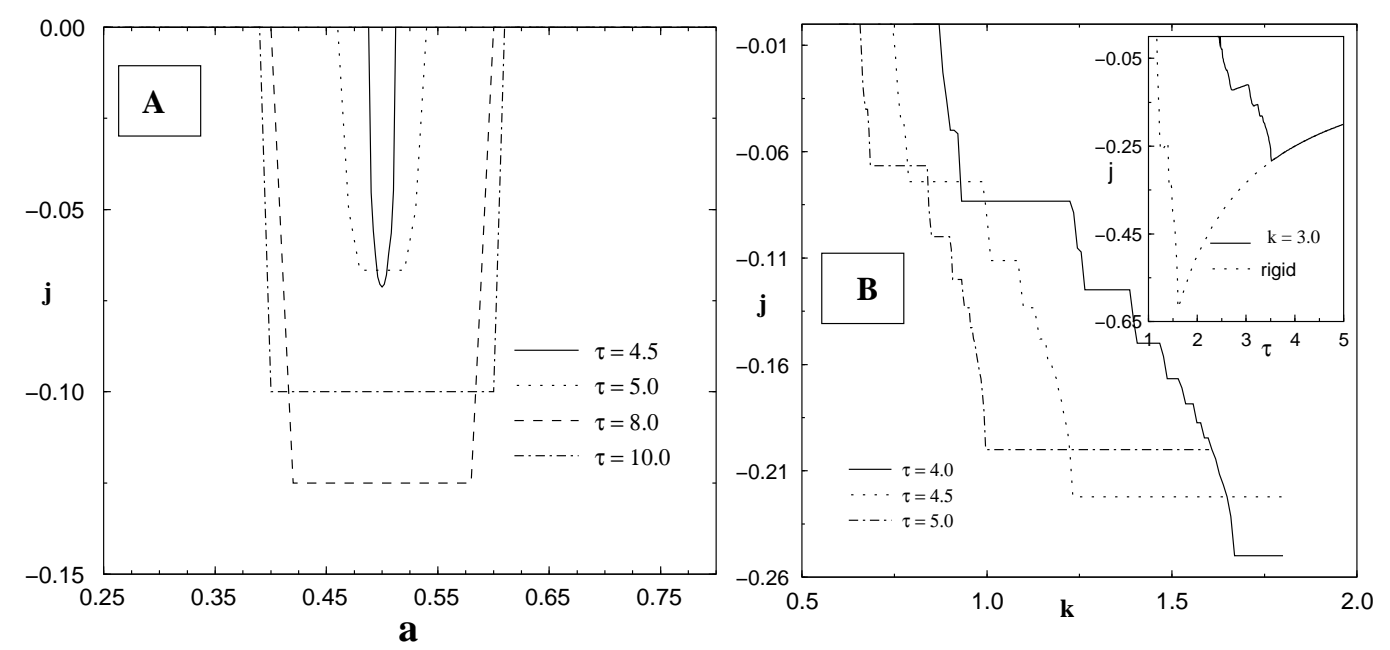

Fig. 1. $[\mathrm{A}]$ Current $j$ vs equilibrium distance $a$ at $k=0.8 ; \quad[\mathrm{B}]$ current $j$ vs coupling constant $k$ for $a=0.5$. The inset shows $j$ vs $\tau$ for same parameter value.

between nonlinearity and external drive.

For large $k$, the current saturates to a constant value, which depends on $\tau$. At these values of $k$, the particles traverse exactly one spatial period in one time period; since our system is overdamped, no multiple hops can occur. The inset shows the variation of $j$ with $\tau$ for $k=3.0$ and $k=\infty$ (rigid rod case). The value of $\tau$ for which current is maximum decreases with increasing $k$.

\subsection{Effect of Noise}

Many of the attributes of the deterministic limit, such as the phase locked behaviour of Fig. (1B), are sensitive to noise. In the presence of thermal fluctuations the noise-induced current exhibits many interesting features. There are three distinct regimes in the parameter space where qualitatively different features are obtained: (i) large $\tau$ and $L_{\min }<a<L_{\max }$, (ii) large $\tau$ and $a<L_{\text {min }}$ and (iii) the small $\tau$ or nonadiabatic regime, which we do not discuss here.

We first study case (i), examining the effect of noise in a parameter range where there is current in the deterministic limit. In the large $\tau, a=0.5$ regime the current $j$ increases initially with $k$ for all values of $D$ as shown in fig.(2A). On further increasing $k$ (not shown) the current saturates after a minimum. The inset shows the variation of $j$ with $D$ for different values of $k$, keeping the other parameters the same. The case $k=0$ is the single particle limit. The location of the peak in the $j-D$ curve is determined by the time taken to diffuse to the basin of attraction of the next well $\left(j_{\max }\right.$ at $\left.D \approx L_{\min }^{2} / \tau=0.16 / 5=0.032\right)$. For non-zero coupling, when one particle 

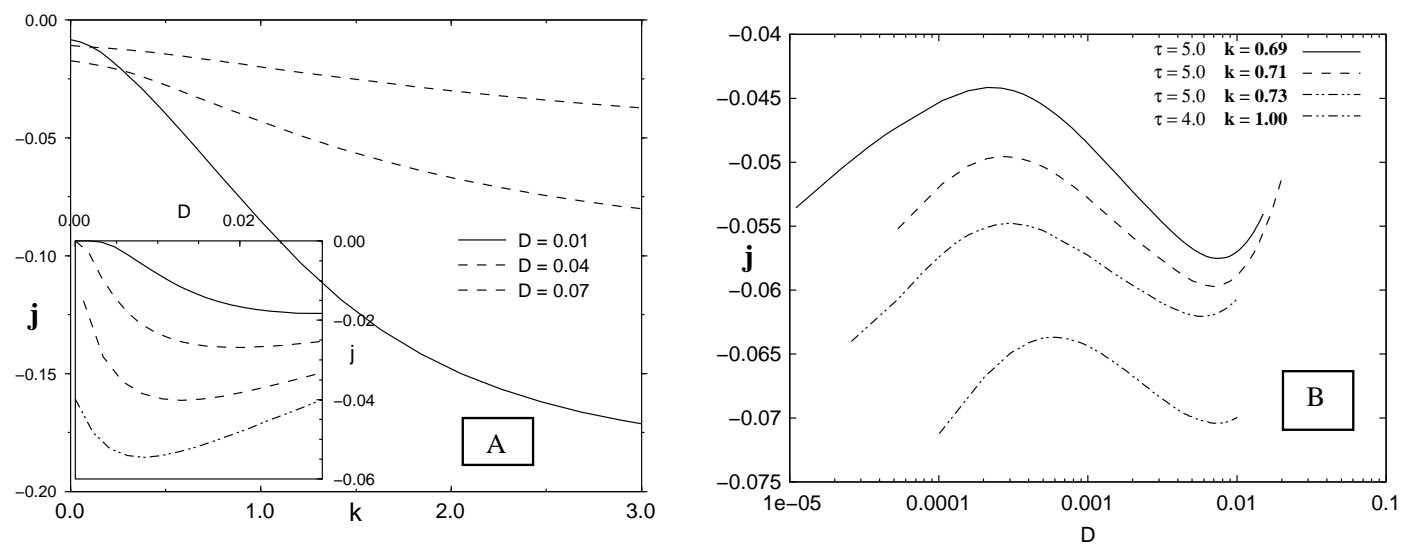

Fig. 2. [A] Current $j$ vs $k$ for $a=0.5$. The inset shows the plot of $j$ vs $D$ for $k=0.0, k=0.3, k=0.5$ and $k=0.67$. ( From top to bottom curve.) [B] The peak in the $j$ vs $D$ ( corresponding to minimum current) emphasizes the noise induced stability phenomena. Here $a=0.5$.

slides down the potential the other is dragged along, thereby enhancing the current. However, on further increasing the noise strength, the potential landscape becomes irrelevant, random hopping in both directions dominates and the absolute value of the current tends to zero, accounting for the minimum seen. At $k=\infty$, the current decreases monotonically with increasing $D$.

Though the increase of current with $D$ for low coupling strength or monotonous decrease at higher $k$ is the generic feature in this regime of operation, for certain values of $k$ (values for which there is phase locking in the deterministic regime, see Fig. (1B)) the current shows highly nonintuitive behaviour. As shown in Fig. (2B), the absolute current initially decreases with increasing $D$, reaches a minima and then again increases with $D$. This feature is reminiscent of the noise induced stability (NIS) of unstable states [12], seen previously in different models, i.e. noise localizes the particle in its basin of attraction. This leads to a decrease in the value of the current with noise. In the high temperature regime, the current should go to zero, explaining, qualitatively, the two extremas seen in the current as a function of noise.

We now investigate the effect of noise in the parameter range where there is no current in the deterministic limit, our case (ii). We work with $a=0.2$. Fig. (3) shows $j$ vs $D$ for various values of $k$. Unlike the previous case where the temperature at which the current peaks decreased with increasing $k$, here this temperature first decreases and then increases with increasing $k$.

The current here arises as a consequence of both the "flashing ratchet mechanism" and the cooperative "pulling effect". For low coupling, the contribution of coupling at a temperature at which the current arising out of the "flashing" mechanism is maximum, is negligible. Hence the net current peaks at a lower value of $T$ compared to the $k=0$ case. For higher $k$, where the current due to coupling dominates, a different scenario obtains. As the stiffness of the spring is larger, a larger fluctuation is required for the particle to transit between wells. The larger the $k$, the stronger the amplitude of 


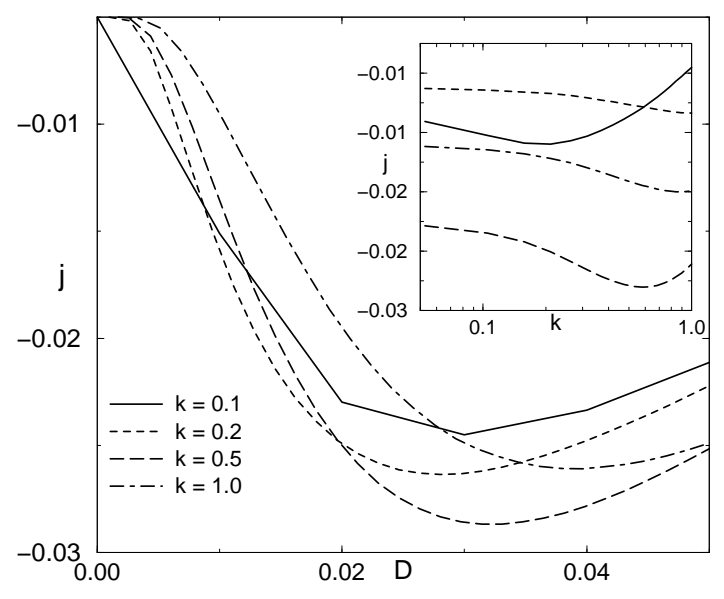

Fig. 3. $j$ vs $D$ for different values of $k, a=0.2$. The inset shows the variation of $j$ vs $k$ in $\log$ scale, for $D=0.01,0.04,0.07$ and 0.1 from top to bottom.

the fluctuation required. If, however, the temperature is too high, significant motion is induced in the opposite direction and the current reaches a maximum. Depending on the parameter regime, the coupling constant may or may not enhance the current compared to the single particle current. This can be clearly seen from Fig. 3 and the inset. This behaviour again differs from the case where there is a finite current in the deterministic limit.

In the nonadiabatic regime obtained for low values of $\tau$, even for extremely small values of $k$, currents may either enhance or decrease relative to the single particle current. This differs from our earlier observation in the adiabatic limit where an enhanced current is obtained for arbitrary small values of $k$, for any $D$. These results will be reported elsewhere.

\section{Conclusions}

Our simple model of a two-headed motor comprised of two coupled Brownian particles in a flashing potential, exhibits unidirectional current. The coupling between particles induces an additional mechanism by which a current may be obtained (via a pulling effect), which is absent in the single particle case[5]. The behaviour of the current depends sensitively on system parameters and exhibits several novel features, such as an enhanced current due to coupling compared to the single particle case, in a regime which we identify. Outside this regime, coupling may or may not lead to a current enhancement.

As a function of noise strength, the current can exhibit noise induced stability in a restricted parameter range. Outside this range, noise initially enhances current and the absolute value of current shows a single maxima. In the deterministic case, we have shown the existence of a window for the two particle separation distance within which there is a deterministic current. This current shows a phase locked behaviour with coupling constant. Unlike 
previous studies on related models, we have not observed current reversals as a function of system parameters.

What relevance, if any, might these results have to real motors? While generically, weakening the coupling of the two heads (particles) appears to reduce the current, we do see specific regimes in which such a reduction leads to an enhancement in the current. As discussed in the Introduction, the increased motility of some Kinesins has been attributed to a larger flexibility of the neck linker. However, whether such simplified models can ever reflect the underlying behaviour of vastly more complex biological systems is unclear.

\section{References}

[1] F. Jülicher, A. Ajdari and J. Prost, Rev. Mod. Phys 69 (1997) 1269.

[2] B. Alberts et. al., Molecular Biology of the Cell, $3^{\text {rd }}$ edition, Garland Publishing (1994).

[3] Y. Aghababaie, G.I. Menon and M. Plischke, Phys. Rev, E, 59, (1999) 2578.

[4] P. Reimann, Physics Reports $\mathbf{3 6 1}$ (2002) 57; A. M. Jayannavar, condmat/0107079

[5] S. Klumpp, A. Mielke, and C. Wald, Phys. Rev. E 63 (2001) 031914; A. Igarashi et al, Phys. Rev. E 64 (2001) 051908.

[6] A. Ajdari, J. Phys. I 4 (1994) 1577.

[7] A. Kallipolitou et. al., EMBO J. 20 (2001) 6226.

[8] Y. H. Song et. al., EMBO J. 20 (2001) 6213.

[9] J. Howard, Nature 389 (1997) 561.

[10] W. Hua, J. Chung, and J. Gelles, Science 295 (2002) 844.

[11] D. Dan, M. C. Mahato and A. M. Jayannavar, Phys. Rev. E 63 (2001) 056307.

[12] R. N. Mantegna and B. Spagnolo, Phys. Rev. Lett 76 (1996) 563; A. Mielke, Phys. Rev. Lett. 84 (2000) 818; N. V. Agudov et al., Phys. Rev. E 64 (2001) 035102. M. C. Mahato, A. M. Jayannavar, Mod. Phys. Lett. B 11 (1997) 815;

D. Dan, M. C. Mahato and A. M. Jayannavar, Phys. Rev. E 60 (1999) 6421.

[13] M. S. Miguel and R. Toral, cond-mat 9707147

[14] H. Risken, The Fokker Planck Equation (Springer Verlag, Berlin , 1984). 